\title{
Characterization and Fabrication of Nanofibers by Electrospinning
}

\author{
Shuxiang Sun ${ }^{\star}$, Xiaoying Huang \\ Henan Vocational College of Applied Technology, Zhengzhou, 450000 China \\ *a465032499@qq.com
}

Keywords: Nonwoven fabric, electrospinning

\begin{abstract}
A kind of nonwoven containing ferulic acid is prepared by electrospinning method. It was observed that the diameter of the fiber was about $200 \mathrm{~nm}$ by SEM. The antibacterial activities against Escherichia coli and $S$. aureus were also investigated. The result showed the nonwoven prepared was effective against Escherichia coli, and S. aureus. Furthermore, Fabric UV transmittance test and air permeability were taken. It was found that the nonwoven achieved good UV protection and air permeability.
\end{abstract}

\section{Introduction}

In recent years, considerable attention has been paid to electrospinning as a versatile technique for producing fibers with diameters ranging from submicron to a few microns. In the typical electrospinning process, nanofibers are produced from an electrostatically driven jet of polymer solution (or melt). The discharged polymer solution jet undergoes a whipping process wherein the solvent evaporates and the highly stretched polymer fiber deposits on a grounded collector. The nonwoven mats of electro spun fibers have shown unique properties, such as dramatically increased surface/volume ratios, excellent mechanical strength, and highly open porous structures [1]. A number of functional nanofibers and composites have been developed through electrospinning by incorporating well-selected functional agents to achieve antibacterial properties. Two common methods are incorporation of antibacterial agents in the electrospinning solutions [2, 3], and electrospinning of polymers with intrinsic antibacterial properties such as queerized chitosan [4]. Heightened awareness of the skin damaging effects of ultraviolet (UV) radiation by the public has led to robust growth in sun and skin care personal product markets. While UV-B (290-320 nm) radiation is principally responsible for sunburn (erythemogenic effect), UV-A (320-400 nm) radiation promotes photo-damage and aging of the skin [5].

Ferulic acid (FA) is a phenolic compound (a member of the cinnamic acid family) found in the most of higher plants. It is generally present in nature as esters with other plant components, such as the hemicelluloses and lignin fractions of the plant cell wall, as well as in suberin and cutin waxy surfaces of leaves and other plant parts. FA is also found esterified to phytosterols present in grain products such as rice bran. As such, FA is a common component of the human diet. It is thus not expected to pose a threat to human health nor to the environment. FA has a strong ultraviolet A (UVA) and B (UVB) absorbance, and it is suitable all-natural replacement for commercially used, petroleumbased sunscreen active ingredients and antioxidants. An obvious use of FA is as a broad UVabsorbing active ingredient in sunscreens, health and beauty aids, and daily-wear cosmetics. Formulation and commercialization of FA into such products are currently being investigated. However, less obvious but important applications also include using FA as an adjuvant to provide natural UV protection in paints, coatings [6].

The aim of this work is to prepare a kind of nonwoven which has the effect of anti-microbial and anti-ultraviolet.

\section{Materials and Methods}

\subsection{Preparation of Nonwoven by Electrospinning}

A power supply (Shanghai Sute Electrical Co., Ltd.) was used to provide voltages in the range of 0-60 kV. To avoid air bubbles spinning solutions were carefully loaded in a $10 \mathrm{~mL}$ syringe fitted with a stainless steel capillary metal-hub needle with an internal inside diameter of $0.5 \mathrm{~mm}$. The positive 
electrode was connected to the needle tip and the grounded electrode to a metal collector wrapped with aluminum foil. The electrospinning process was carried out at room temperature and relative humidity $68 \pm 3 \%$. A fixed electrical potential of $15 \mathrm{kV}$ was applied across a fixed distance $(12 \mathrm{~cm})$ between the tip and the collector. The feed rate of solutions was controlled at $2.0 \mathrm{ml} \cdot \mathrm{h}^{-1}$ by mean of a single syringe pump (Cole-Parmer, USA). The solution was prepared by dissolving $0.05 \mathrm{~g}$ FA and $0.95 \mathrm{~g}$ PAN in $10 \mathrm{~mL}$ DMAC. The formed nonwoven membranes were allowed to be further dried for over $24 \mathrm{~h}$ at $40{ }^{\circ} \mathrm{C}$ and under vacuum (320 Pa) in a DZF-6050 Electric Vacuum Drying Oven (Shanghai Laboratory Instrument Work Co. Ltd., Shanghai, China) to remove of residual organic solvent.

\subsection{Morphology}

The surface morphology of the electro spun fibers were assessed using a JSM-5600LV scanning electron microscope (Electron Optics Laboratory Co. Ltd., Japan). Prior to the examination, samples were gold sputter-coated under argon to render them electrically conductive. Electron micrographs were then taken at an excitation voltage of $15 \mathrm{kV}$.

\subsection{Determination of Antibacterial Activity}

S. aureus was incubated in $10 \mathrm{~mL}$ of a $3.1 \%$ yeast-dextrose broth (containing $10 \mathrm{~g} / \mathrm{L}$ peptone, 8 $\mathrm{g} / \mathrm{L}$ beef extract, $5 \mathrm{~g} / \mathrm{L}$ sodium chloride, $5 \mathrm{~g} / \mathrm{L}$ glucose, and $3 \mathrm{~g} / \mathrm{L}$ yeast extract at a $\mathrm{pH} 6.8$ ) for $6-8 \mathrm{~h}$ at $37^{\circ} \mathrm{C}$ until the exponential growth phase was reached[8]. The bacteria-containing broth was centrifuged at $3000 \mathrm{rpm}$ for $10 \mathrm{~min}$, and after removal of supernatant, the cells were washed twice with sterile phosphate-buffered solution (PBS). The bacteria cells were suspended to provide a final density of 106 cells $/ \mathrm{mL}$ in PBS (based on standard calibration with the assumption that the optical density of 1.0 at $540 \mathrm{~nm}$ is equivalent to approximately 109 cells $/ \mathrm{mL}$ ) [9].

\subsection{Determination of Effect of Anti-ultraviolet}

Fabric UV transmittance test was taken by UV-1000F Ultraviolet Transmittance Analyzer (America Lab sphere Co. Ltd.). The optical design meets the requirements of the USA standards AATCC183-1998.

\subsection{Determination of Air Permeability of the Nonwoven}

Air permeability of cotton fabrics was measured, according to GB5453-85, Standard Test Method for Air Permeability of Textile Fabrics, using a Y561 Air Permeability Tester (China).

\section{Results and Discussion}

\subsection{Morphology}

The spinning solution was a transparent bright yellow this being due to the complete solubility of FA in PAN when co-dissolved in DMAC. The configuration of the fibers is shown in Fig. 1.The diameter of the fibers is about $200 \mathrm{~nm}$. The interspaces between fibers distributed uniformly. Fig. 2 is the photograph of the nonwoven taken by digital camera. The surface of the nonwoven is very smooth and dense.

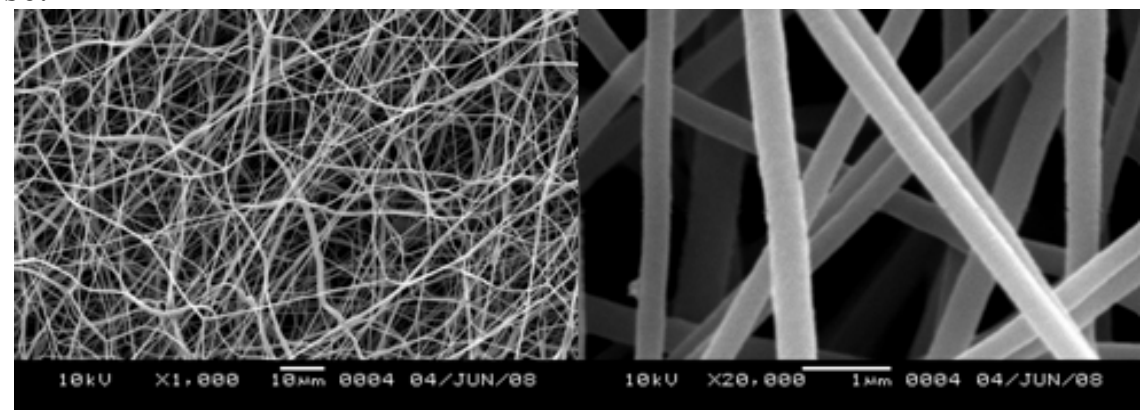

Fig. 1 SEM photograph of electro spun PAN fibers containing 5\% FA. 


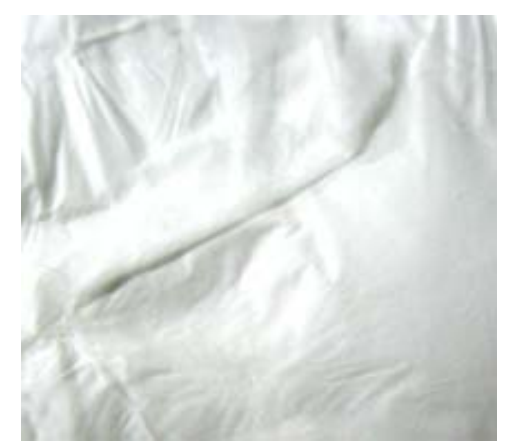

Fig. 2 Photograph of electro spun nonwoven.

\subsection{Antibacterial Activity}

Antibacterial efficacy of surface modified nonwoven containing FA was investigated by estimating the number of viable bacteria cells in the $\mathrm{S}$. aureus and E. coli suspension after being in contact with nonwoven for various periods of time, and the results were shown in Fig. 3.

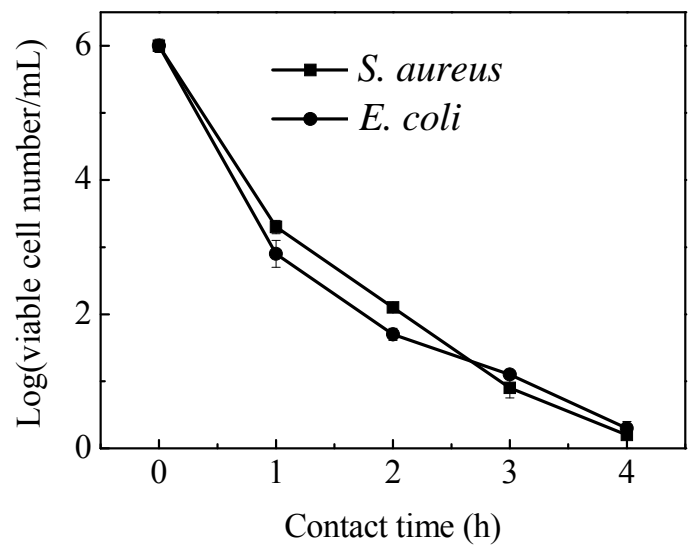

Fig. 3 Viable cell numbers of Staphylococcus aureus and Escherichia coli as a function time in contact with: nonwoven. The cell number was determined by surface spread plate method.

\subsection{Effect of Anti-ultraviolet}

Because the elevated exposure of human skin to UV radiation of the solar spectrum leads to more serious sunburns, premature skin aging, allergies and even skin cancer, the protection from excessive UV irradiation becomes a key requirement for skin-care clothing [10]. Generally, a UPF value of 40$50+$ indicates an excellent protection of human skin from UV damage [11]. When the thickness of nonwoven reach above $100 \mu \mathrm{m}$, the nonwoven have achieved good UV protection.

Table 1. The anti-uv effect of nonwoven fabric

\begin{tabular}{ccc}
\hline Thickness of nonwoven $(\boldsymbol{\mu m})$ & UPF value & Effect of protection \\
\hline 50 & 21.3 & Good \\
100 & 40.5 & Very good \\
200 & 52.6 & Extremely good \\
400 & 119.7 & Extremely good \\
\hline
\end{tabular}

\subsection{Air Permeability}

Air permeability is one of the major properties of textile materials and is governed by factors like the fabric structure, density, thickness, and surface characteristics etc. The air permeability of nonwoven with different thickness was examined (Table .2). By increasing the thickness of nonwoven from $50 \mu \mathrm{m}$ to $400 \mu \mathrm{m}$, the air permeability was reduced.

Table 2. The air permeability of nonwoven fabric.

\begin{tabular}{cc}
\hline Thickness of nonwoven $(\boldsymbol{\mu m})$ & Air permeability $\left(\mathbf{c m}^{3} / \mathbf{s} / \mathbf{c m}^{2}\right)$ \\
\hline 50 & 128.6 \\
100 & 105.4 \\
200 & 80.7 \\
400 & 57.6 \\
\hline
\end{tabular}




\section{Conclusion}

In summary, when FA were conveniently applied to fabric by electrospinning, the resulting nonwoven can largely reduce UV transmission, and possess highly effective antibacterial activities. This novel nonwoven would be useful in building a next generation of health-care clothing system that will promote wearers' comfort and skin health.

\section{References}

[1]. Y. Chen, X.S. Li, K.G. Neoh, Z.L.Shi, E.T. Kang, Surface modification and antibacterial activity of electrospun polyurethane fibrous membranes with quaternary ammonium moieties, $\mathrm{J}$. Membrane Sci. 320 (2008) 259-267.

[2]. Melaiye, Z.H. Sun, K. Hindi, A. Milsted, D. Ely, D.H. Reneker, C.A. Tessier, W.J. Youngs, Silver(I)-imidazole cyclophane gem-diol complexes encapsulated by electrospun eecophilic nanofibers: formation of nanosilver particles and antimicrobial activity, J. Am. Chem. Soc. 127 (2005) 2285-2291.

[3]. W.J. Jin, H.J. Jeon, J.H. Kim, J.H. Youk, A study on the preparation of poly(vinylalcohol) nanofibers containing silver nanoparticles, Synth. Met. 157 (2007) 454-459.

[4]. M. Ignatova, K. Starbova, N. Markova, N. Manolova, I. Rashkov, Electrospun nano-fibre mats with antibacterial properties from quaternised chitosan and poly(vinyl alcohol), Carbohydr. Res. 341 (2006) 2098-2107.

[5]. J.A. Laszlo, D.L. Compton, F.J. Eller, S.L. Taylor, T.A. Isbell, Packed-bed bioreactor synthesis of feruloylated monoacyl- and diacylglycerols: clean production of a "green" sunscreen, Green Chem. 5 (2003) 382-386.

[6]. D.L. Comptona, J.A. Kenar, J.A. Laszlo, F.C. Felker, Starch-encapsulated, soy-based, ultraviolet-absorbing composites with feruloylated monoacyl- and diacylglycerol lipids, Ind. Crops Prod. 25 (2007) 17-23.

[7]. J. Zeng, X.S. Chen, X.B. Jing, Ultrafine fibers electrospun from biodegradable polymers, J. Appl. Polym. Sci. 89 (2003) 1085-1092.

[8]. D. Cunliffe, C.A. Smart, C. Alexander, E.N. Vulfson, Bacterial adhesion at synthetic surfaces, Appl. Environ. Microbiol. 65 (1999) 4995-5002.

[9]. H. Hogt, J. Dankert, J. Feijen, Adhesion of coagulase-negative staphylococci to methacrylate polymers and copolymers, J. Biomed. Mater. Res. 20 (1986) 533-545.

[10]. B. Fei, H.F. Lu, K.H. Qi, H.F. Shi, T.X. Liu, X.Z. Li, J.H. Xin, Multi-functional microcapsules produced by aerosol reaction, Aerosol Sci. Technol. 39 (2008) 1089-1098.

[11]. AS/NZS 4399: 1996. Sun protective clothing-evaluation and classification (1996). 